University of Nebraska - Lincoln

DigitalCommons@University of Nebraska - Lincoln

Robert G. Fuller Publications and Presentations Research Papers in Physics and Astronomy

April 1994

\title{
TEACHING AND LEARNING PHYSICS WITH INTERACTIVE VIDEO
}

Dean Zollman

Kansas State University, Manhattan, Kansas

Robert Fuller

rfuller@neb.rr.com

Follow this and additional works at: https://digitalcommons.unl.edu/physicsfuller

Part of the Physics Commons

Zollman, Dean and Fuller, Robert, "TEACHING AND LEARNING PHYSICS WITH INTERACTIVE VIDEO" (1994). Robert G. Fuller Publications and Presentations. 35.

https://digitalcommons.unl.edu/physicsfuller/35

This Article is brought to you for free and open access by the Research Papers in Physics and Astronomy at DigitalCommons@University of Nebraska - Lincoln. It has been accepted for inclusion in Robert G. Fuller Publications and Presentations by an authorized administrator of DigitalCommons@University of Nebraska - Lincoln. 


\section{TEACHING AND LEARNING PHYSICS WITH INTERACTIVE VIDEO}

\section{Using computer-controlled digital video, students can view and analyze the physics of events as common as the snapping of a towel, as graceful as the motion of a dancer or as extraordinary as the collapse of the Tacoma Narrows Bridge.}

\author{
Dean A. Zollman and Robert G. Fuller
}

Dean Zollman is a professor of physics at Kansas State University, in Manhattan, Kansas. Robert Fuller is a professor of physics at the University of Nebraska, Lincoln.
In 1961 the psychologist Jerome Bruner concluded a discussion of innovative teaching materials of the late 1950 s by saying, "The intelligent use of [audiovisual] resources will depend upon how well we are able to integrate the technique of the filmmaker or the program producer with the technique and wisdom of the skillful teacher." 1 The Physical Sciences Study Committee had just completed a series of films that attempted to bring together current film technology, the expertise of the film producer and the knowledge and experience of outstanding physics teachers. Today a critic of pedagogical films would find much to change in the PSSC films. Yet they did open new territory for physics teachers, and parts of these films survive today in the videodisc series Physics: Cinema Classics. ${ }^{2}$

Physics teachers have long used visual media to teach. Well before any form of motion picture was available, lecture demonstrations provided visualizations of the basic concepts of physics. Video and films for physics teaching date from at least the early $1950 \mathrm{~s}$, when the American Association of Physics Teachers sponsored a set of films, which have since been lost. Harvey White's televised physics programs date from the latter half of the 1950s. The PSSC films and the Super-8 film loops produced by Franklin Miller, Project Physics and others became important tools in physics teachers' collections in the 1960 s.

But while film and videotape can in principle bring some of the knowledge and experience of outstanding teachers into any classroom, they have a major limitation. The control exercised by the classroom teacher or the student is limited to turning the film on or off. Once the film or videotape is turned on, the flow of information is controlled by the film production team. Thus one of the most important capabilities of the experienced teacherthe ability to respond immediately and appropriately to the needs of the students-is not available in these media.

Interactive video gives teachers a variety of audiovisual materials and techniques that they can control. Should teachers wish, they can pass that control to 

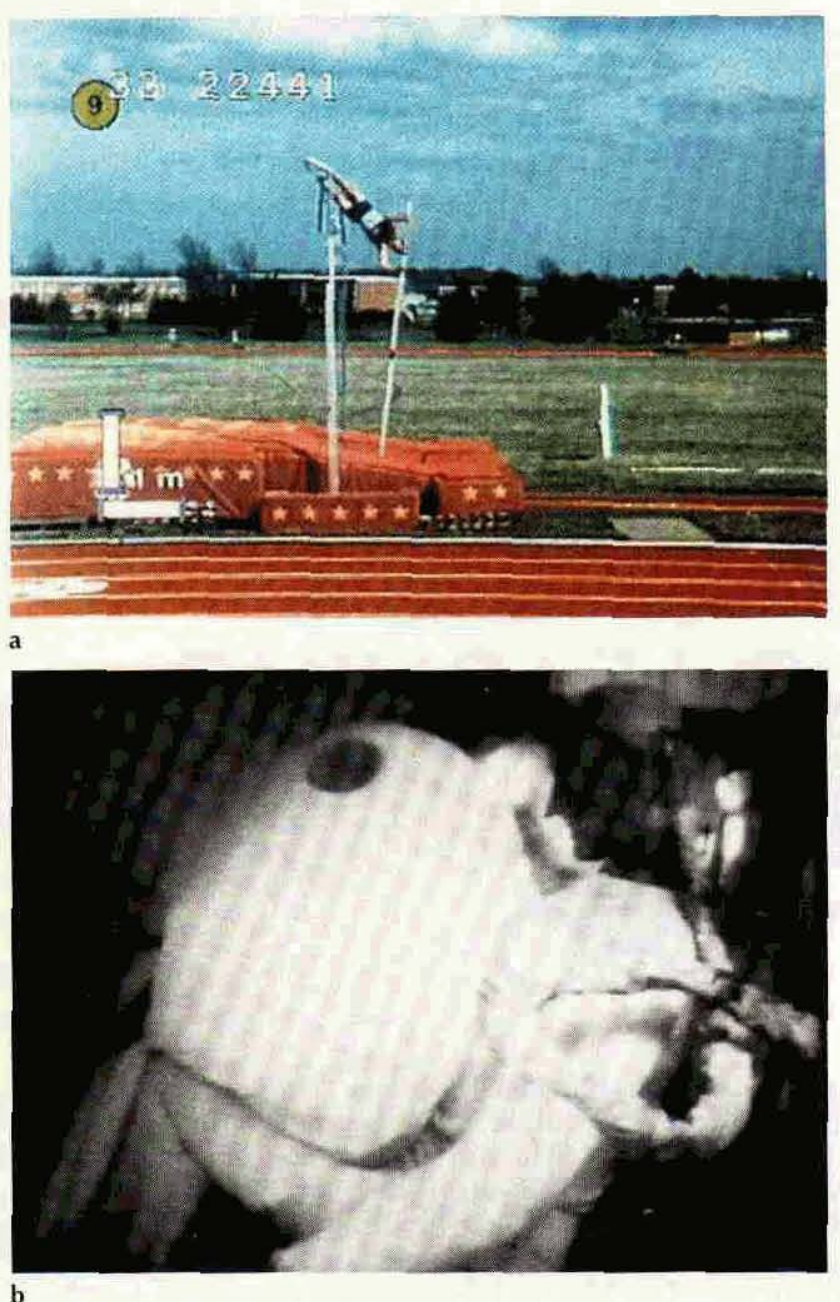

b

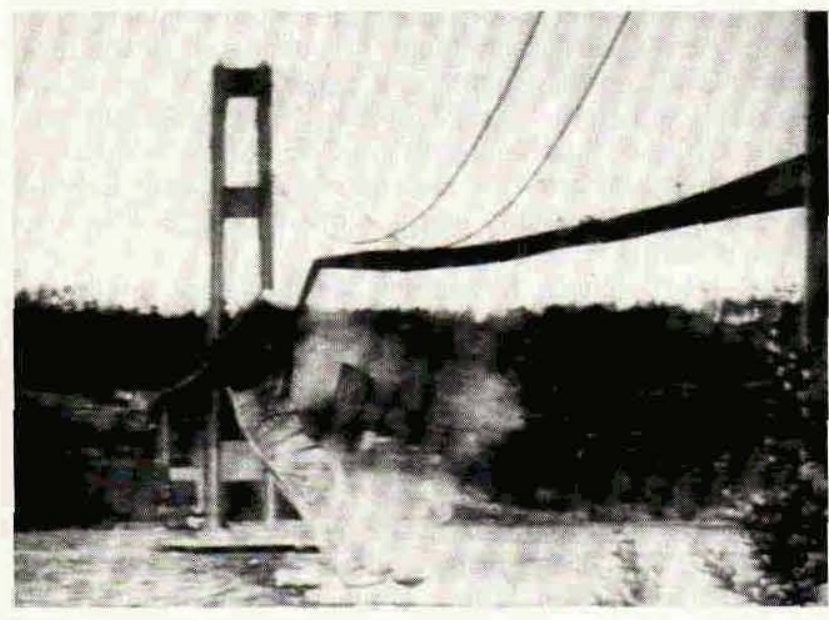

c

the students and so enable them to answer their own inquiries at a pace that is comfortable for them. In many ways interactive video makes physics teachers a part of the media production and instructional design team even though they never visit a television studio.

\section{What is interactive video?}

In a generic sense interactive video is any video in which the user has more than minimal on-off control over what appears on the video screen, including:

$\triangleright$ random access, which allows the user to select and display a segment or an individual frame (picture) with minimal search time
Interactive video makes possible the detailed study of real-life events such as the motion of a pole-vaulter (a), the motion of a dummy during an auto crash (b) and the collapse of the Tacoma Narrows Bridge (c). Figure 1 $\triangleright$ still frame, which allows any frame to be clearly displayed for as long as the user wishes to view it

$\triangleright$ step frame, which enables the user to display the next or previous frame

$\triangleright$ slow play, which lets the user play the video at any speed up to real-time either forward or backward. (Realtime projection speed is 30 frames per second in North America and Japan and 25 frames per second in most of the rest of the world.)

The videodisc makes this level of control possible. A random-access videodisc contains up to 54000 numbered frames of analog video and two independent channels of audio. Using the search mechanism of the videodisc player, one can quickly reach any frame. Because the player uses laser technology to read the information, the quality of the image and audio signals is not degraded if the viewer watches one frame for a long time. (Videotape cannot provide truly interactive video, because an individual frame degrades if it is displayed for a long time and because random access is difficult.)

For this type of interactivity to be useful, students and teachers must be able to control the videodisc player easily. At present, three primary methods of control exist:

$\triangle$ Keypads are similar to the remote control for a videocassette recorder. They allow the user to enter all of the functions described above.

$\triangleright$ Bar-code readers allow the user to control the videodisc player by means of information stored in bar codes. $\triangleright$ Computer control delivers all the commands needed for full interactivity through an RS-232 serial port that exists on many videodisc players.

A computer-controlled videodisc player is a basic element of a so-called hypermedia system for learning physics. ${ }^{3}$ Various groups have used programming languages and popular authoring systems such as Hypercard on the Apple Macintosh and Toolbook on MS-DOS machines to control videodisc players. (See the article by Jack M. Wilson and Edward F. Redish in PHYSICS TODAY, January 1989, page 34 .)

We have been exploring the use of interactive video in physics teaching since 1978. The approaches we have developed range from simple motivation of the study of a topic to detailed analysis of complex real-world events using mathematical and visual models.

\section{Motivation}

Showing the connection between physics and events outside the classroom helps students perceive the relevance of physics to their lives and how physical laws help them to understand nature. The events can be rather ordinary, such as the motion of a pole-vaulter or high jumper; ${ }^{4}$ they can be more unusual events, such as the motion of a 


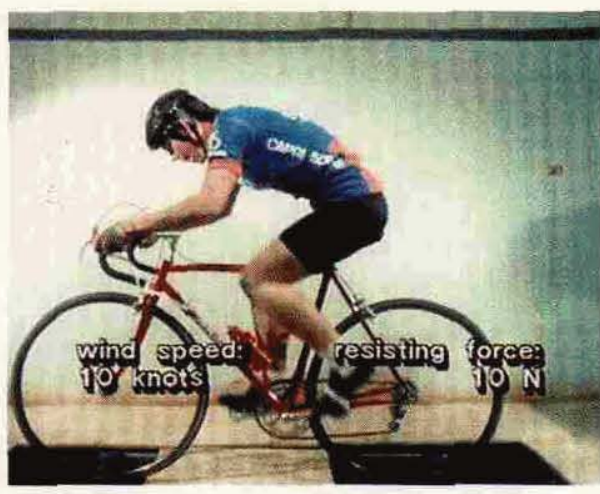

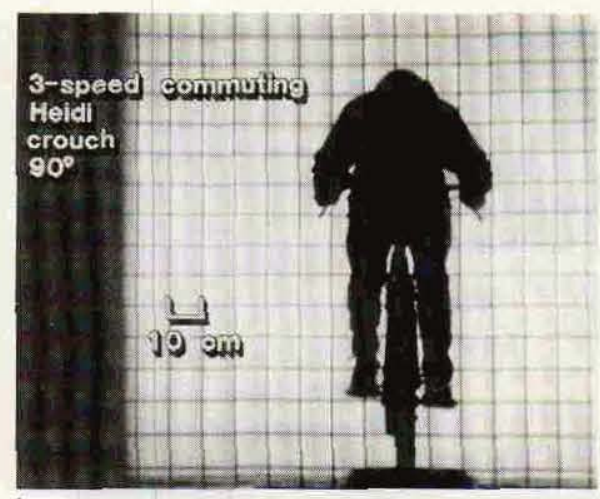

b
Wind resistance on a bicyclist in a wind tunnel can be studied with interactive video. a: Wind speed and drag force are shown along with a side view of the cyclist. b: A grid superimposed on a frontal view of the cyclist (Heidi) in the given cycling position (crouch, $90^{\circ}$ ) permits students to estimate the surface area the cyclist presents to the wind in this position. Figure 2 mannequin as it strikes an air bag during a car collision; ${ }^{5}$ or they can be extraordinary occurrences, such as the collapse of the Tacoma Narrows Bridge. ${ }^{6}$ (See figure 1.) Strictly speaking, these events could be shown on videotape rather than on interactive video. However, an interactive video format can enrich classroom discussion by giving the teacher the ability to replay any portion of the video and alter the playing speed in response to student questions. For example, a teacher can return quickly to the frame that shows the high jumper just as she clears the bar and then step through the video sequence frame by frame while discussing the location of her center of mass. The students can watch the video at any speed they desire and observe the details of an event. By giving students some control over the images they see and how they analyze and apply physics to those images, interactive video can enhance the students' motivation to study physics.

Motivation can be further enhanced when such scenes are shown before the students begin to study a topic, as an introduction to it. For example, when students watch a video of the collapse of the Tacoma Narrows Bridge, usually their immediate response is, "The wind must have been blowing really fast." One can then return to a portion of the video that shows that the wind did not uproot trees along the shore even though it destroyed the bridge. When a student asks about the relationship between wind speed and amplitude of oscillations, one can select and play a portion of the audio in which one of the bridge's engineers discusses that topic. Thus in seeking to understand why the bridge collapsed, students can become interested in studying wave motion and forced oscillations.

\section{Interactive video as a laboratory tool}

Interactive video also provides a means by which students can collect, analyze and model data from events outside the classroom. Sometimes they collect the data by reading instruments recorded in the original scene. More often, however, students collect data in a manner similar to that used by a researcher who analyzes the recorded events.

For parts of the videodise Energy Transformations Featuring the Bicycle, ${ }^{7}$ we recorded instruments showing the wind speed and force of the wind on cyclists riding

A classic film from the series produced by the Physical Sciences Study Committee shows two balls falling-one with a horizontal initial velocity. When it was transferred to videodisc, ${ }^{2}$ a clock marked in 24 ths of a second and a distance indicator were added. Figure 3 bicycles in a wind tunnel. These data are displayed on the screen for different positions of the cyclist. Elsewhere on the disc, a frontal view of the cyclists in each of their cycling positions is superimposed on a grid so that students can estimate the surface area each cyclist presents to the wind. (See figure 2.) Using a catalog of events, students can select which wind speeds or riding positions they wish to analyze and determine how the force on the cyclist varies with wind speed and surface area.

Frequently the students can collect distance, time and other data directly from a video scene, much as they would in an actual laboratory experiment. For example, a scene from a PSSC film reproduced on the videodisc Physics: Cinema Classics shows two balls. (See figure 3.) One ball is dropped at the same moment that the other is projected horizontally. Students can step the video forward one frame at a time and measure the vertical and horizontal distances traveled by each ball. They can compare the vertical accelerations of the two balls and, knowing the scale on the screen and the time between pictures, calculate the acceleration.

Similar techniques can be applied to more complex events that cannot be created in an instructional laboratory. For example, the videodisc Physics of Sports ${ }^{4}$ provides a variety of scenes from which students can collect data, including a comparison of a distance runner with a sprinter and a high-speed film of an arrow as it leaves a bow. The videodisc Physics and Automobile Collisions ${ }^{5}$ features scenes of automobiles colliding with walls and each other at film speeds up to 500 frames per second. (See figure 4.)

In one of the most popular techniques for collecting such data, students place a sheet of clear acetate on the video screen, identify an object to provide a distance scale

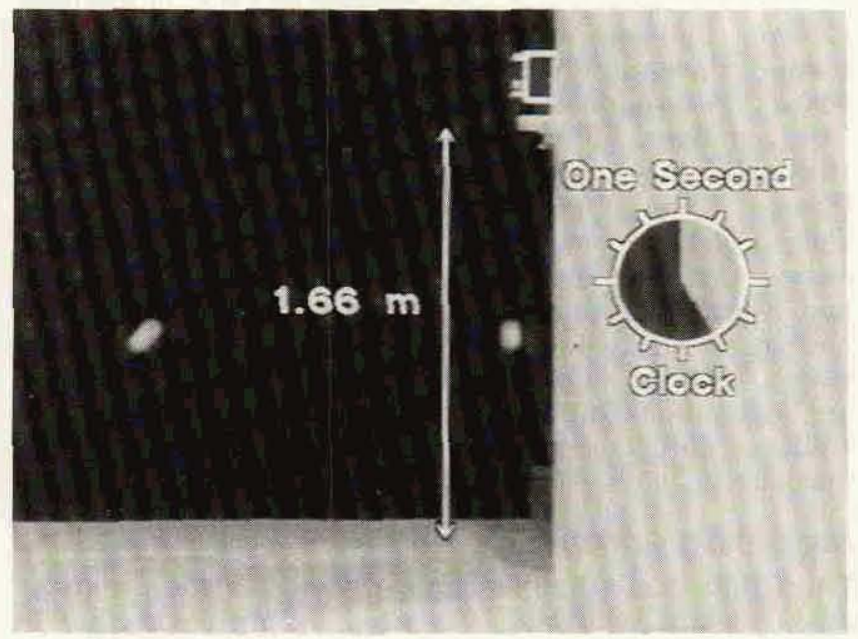




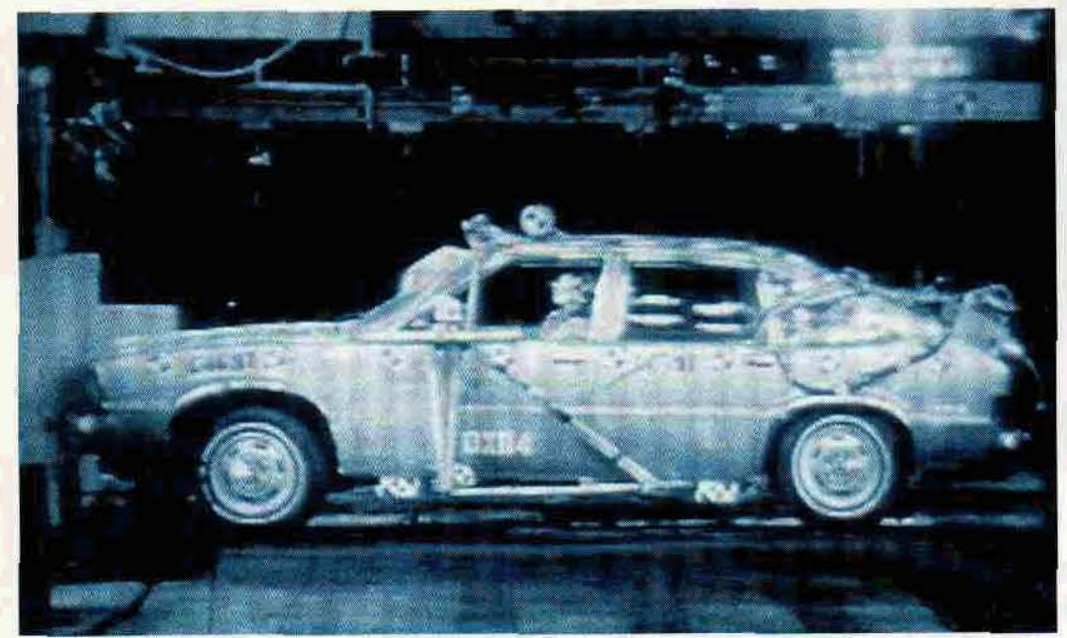

High-speed automobile collision with a wall (a) and a plot of the distance-time data collected from the video images (b). Figure 4

a

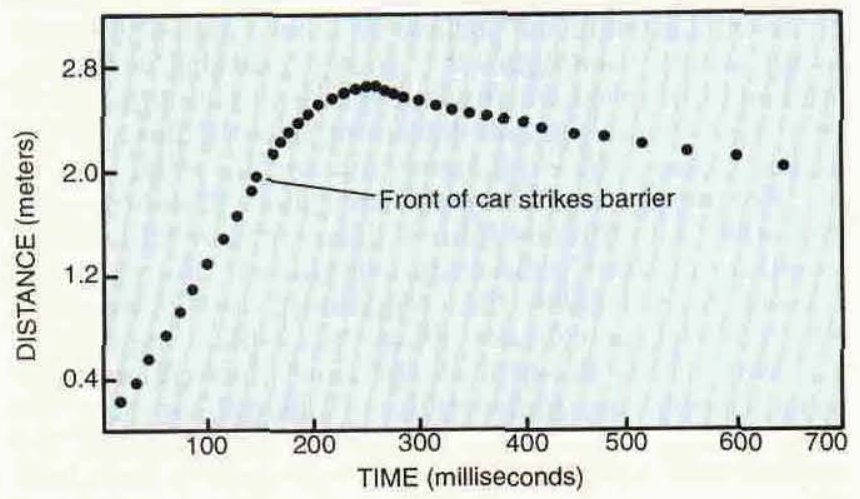

and then mark points of interest in the picture. As they step the video forward, they mark those points again in each frame until the end of the scene. (See the cover of this issue.) This procedure requires only a short time, so many students can use a single videodisc player during a regular class period. Once the points are marked on the acetate, it is removed from the screen and laid on a sheet of graph paper, from which the students read off coordinates for the measurement scale and for each of the points. Knowing the time and distance separations, they can calculate standard kinematic variables, such as velocities and accelerations. If the masses of the objects are known, the students can compute forces, energies and momenta. They thus can use the laws of physics to explain natural phenomena.

A similar but more sophisticated method of analysis involves the use of a computer that can superimpose computer graphics on a digitized video scene. Computer boards are available for both MS-DOS and Apple Macintosh computers to accomplish this task. Because many videodisc players can be controlled by the computer through a serial port, students can control the video. Once a picture is on the video screen, students move the computer cursor to the location of interest and then read and record coordinates directly into a file for later use and analysis. The interactive nature of the software allows teachers to control many aspects of the students' analysis. For example, if the teacher decides that the students do not need to calculate the scaling factor, the teacher can have the computer do it automatically.

\section{Modeling complex events}

The creation of simplified models for complex events is ar important analytical tool used by physicists that is often not treated in detail in standard instructional laboratory experiments, since they are simplified by their very nature. Interactive video, however, can present events that require simplification for students (or physicists) to understand them. The videodiscs Physics of Sports and Studies in Motion ${ }^{8}$ show a number of athletic and dance events that allow students to use simplified visual models of human motion. Following the techniques used by research kinesiologists, we ask the students to draw stick figures on top of a digitized video image of, for example, a high jumper. Figure 5 depicts three simple models for the human body used in this exercise. The first model simply places the center of mass of the person at the hip, independent of the person's orientation. The second is a three-segment model, with one segment for the arms and a "hinge" at the hip. The third is a five-segment model. (A careful study in kinesiology or the analysis of sports will include more than 20 such segments.) We ask students to use these models as best they can to draw the position of the jumper during the event. Then, using mass distribution data from cadaver studies and assuming that each segment has a uniform mass distribution, they can calculate the center of mass for each model. Such visual models and simple calculations may help students discover that the center of mass of a high jumper or pole-vaulter goes under the bar rather than over it or that a dancer manipulates his or her body to look as if it is floating even though its center of mass undergoes parabolic motion. $^{10}$ (See the article by Kenneth Laws in PHYSICS TODAY, February 1985, page 24.)

One can also do mathematical modeling with data collected from an interactive video sequence. For example, the data from figure 4 can be described rather accurately by a model in which the car has a constant velocity before contact with the wall, a constant negative acceleration while in contact with the wall and a different constant velocity after leaving the wall. Since the car was pulled by a cable until it struck the wall, the constant velocity before collision is not a surprise and in fact is a test of our students' data-taking ability. However, given the complexity of the interaction between the car and the wall, a constant acceleration for the period of contact between the car and the wall is a little surprising.

A mathematical analysis that requires more sophistication involves the motion of an arrow being shot by a compound bow, a complicated device that applies a varying force to the arrow. Two scenes on the Physics of Sports videodisc let students compare the work done on the arrow with the kinetic energy of the arrow as it leaves the bow. First a spring scale is used to pull the bow back and then gradually allow it to move forward. In the 
Motion of a high jumper is analyzed using three different models: a point-mass model (a), a three-segment model (b) and a five-segment model (c). Figure 5

second scene, an archer shoots an arrow. (See figure 6.) From the scene with the archer, students can measure the maximum displacement of the bowstring from its equilibrium position and the arrow's velocity as it leaves the bow. Then they can use the spring scale scene to determine the force at each point from the string's equilibrium position to its maximum displacement. Because the force on the arrow is not constant, students must determine a sensible mathematical model for the force and numerically integrate it to calculate the work done.

\section{Qualitative observations}

Many physics teachers seek to have their students simply observe their own world more carefully. Interactive video provides a training ground for doing so. By freezing scenes and looking at the physics of an event more carefully, ${ }^{11}$ students can learn to control variables. In our lesson using the videodisc The Puzzle of the Tacoma Narrows Bridge Collapse, we ask students to determine whether a steady or periodic force of the wind is more likely to cause the type of oscillating pattern observed and whether the frequency of the force is an important variable. ${ }^{12}$ To allow the students freedom to investigate this question in their own manner, we created in the TV studio a model of the bridge and filmed its interaction with a "wind" provided by a standard three-speed window fan. In addition to steady winds from the fan, our colleague Tom Campbell imitated pulsating forces of two different frequencies for each wind speed by chopping the flow of air with a piece of cardboard. (See figure 7.) The video screen shows students a three-by-three matrix of these nine events. They can watch the events in any order and draw qualitative conclusions about whether wind speed or pulsating force was an important factor in the demise of the Tacoma Narrows Bridge.

Another scene, on the videodisc Physics: Cinema Classics, combines two films to help students determine the nature of the interaction between an alpha particle and a nucleus. A Rutherford scattering simulation allows students to qualitatively trace out the trajectories of simulated alpha particles. They can then compare the alpha trajectories with a film of the paths on an air table of two hard spheres, two magnetic objects and two electrically charged objects to understand why Rutherford drew some of the conclusions he did about the nature of the atom.

\section{Video as a laboratory probe}

While we have developed a number of techniques and videodiscs for interactive video laboratory exercises, we have only recently asked students to analyze videos of
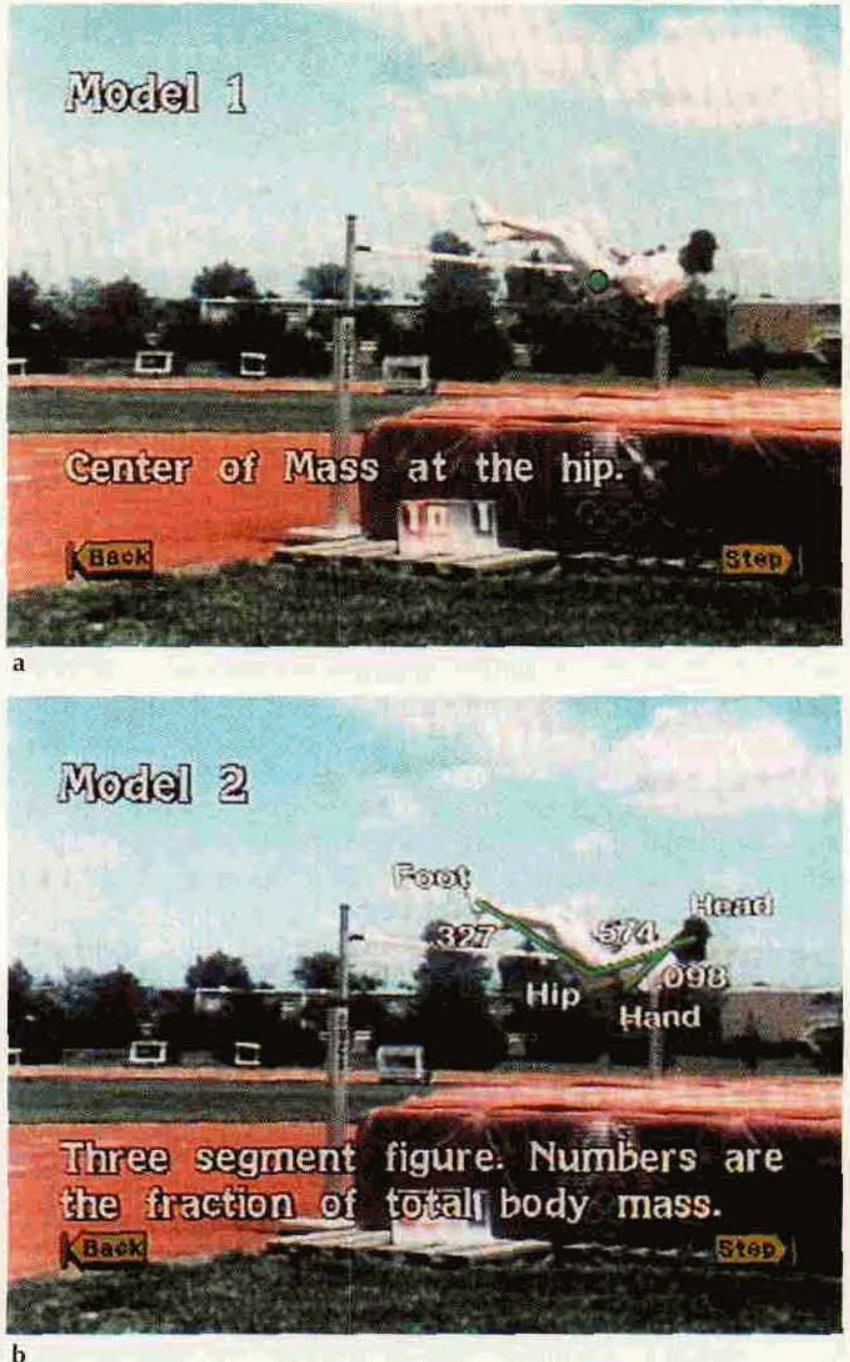

b

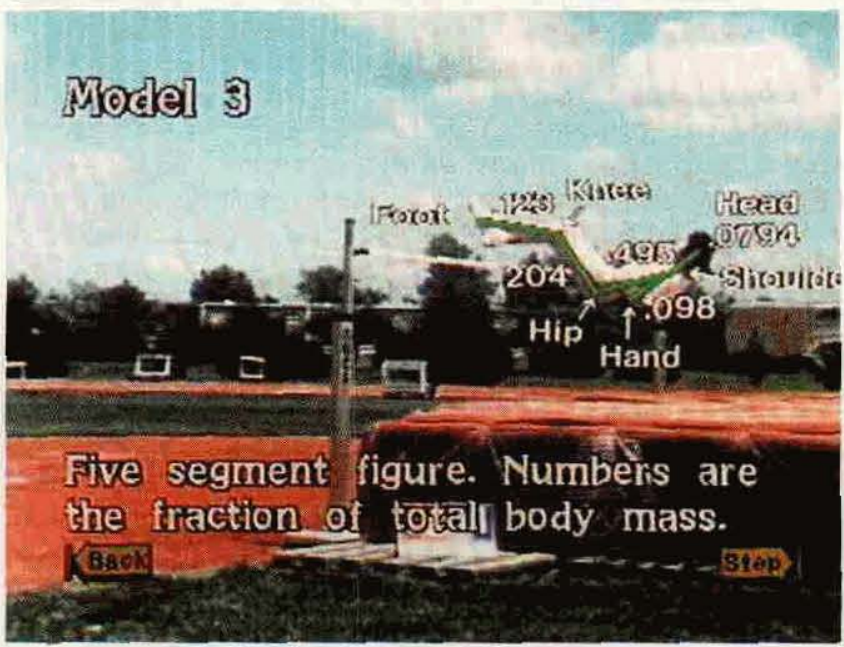

their own making. Students can in principle collect data on videotape and analyze the motion using the techniques described above. ${ }^{13}$ Unfortunately, until very recently good single-frame viewing and step forward and reverse functions were not available on low-cost VCRs. However, the ability to use a computer to capture a still picture from a videotape has been available for several years.

Robert J. Beichner ${ }^{14}$ and Loren M. Winters ${ }^{15}$ exploited this capability in quite different ways. Beichner's students captured a series of still images from videodisc 
or videotape and then used software to collect positiontime data from these stills. Winters developed a unique combination of high-speed stroboscopic flashlamps and still-image capture: In a dark room up to four computercontrolled flash units were fired in rapid succession, and the images created by the flashes were captured as a single digitized image. Winters's students have used this technique to investigate very rapid motion such as the movement of the end of a towel being snapped.

Another approach to collecting digital data from an analog video was developed several years ago by three groups working independently. ${ }^{16}$ Using a computer equipped with a special board, each of these groups collected two-dimensional data only about the area on the screen where an event was imaged. (Collection of three-dimensional data requires two cameras and two computer boards.) Collecting data only about this limited region on the video screen alleviated the memory limitations that until recently made computer video impractical. Data analysis programs provided the students with a variety of derived quantities such as velocity and acceleration. However, the need for the specialized computer boards limited the use of this technique to a few institutions.

The use of video in educational laboratories ${ }^{17}$ received a boost in recent years with the introduction of low-cost video digitizing boards. This hardware enables a user to connect any video source to the computer, digitize the incoming analog signal and store the resulting digital information on a computer disk. This technique is relatively new, and at present no industry standard exists for the format of the video or the hardware on which it is played. The most popular packages for digitizing video are Video for Windows, for computers using MS-DOS, and Quicktime and Digital Video Interactive, for both Apple Macintosh and MS-DOS machines. These packages differ in the compression techniques that enable one to store video on a hard drive as well as in the computer platform used.

Several investigators are developing techniques for using digitized video in a laboratory setting. ${ }^{18}$ While details of the approaches differ, they share several components. The students first record experiments with a video camera or capture existing video from a tape or disc. Next they look at the video one frame at a time, move the cursor to points of interest and collect positiontime data. Analysis is completed using programs written for this approach or, more commonly, productivity software such as a spreadsheet program. (See the article by Priscilla W. Laws in PHYSICS TODAY, December 1991, page 24.) The use of this technique is spreading rapidly and holds much promise for helping students visualize the concepts of physics.

This approach to instructional laboratories can be used for analyses beyond the traditional two-dimensional kinematic studies. Under our supervision, students have analyzed motion from different moving reference frames by mounting a video camera on a cart and capturing the video on the computer's hard drive.

Because the video information is in digital rather

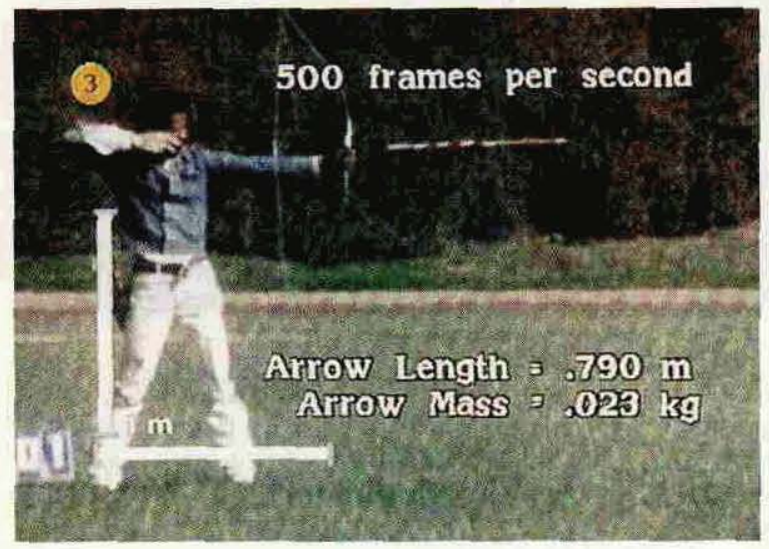

a

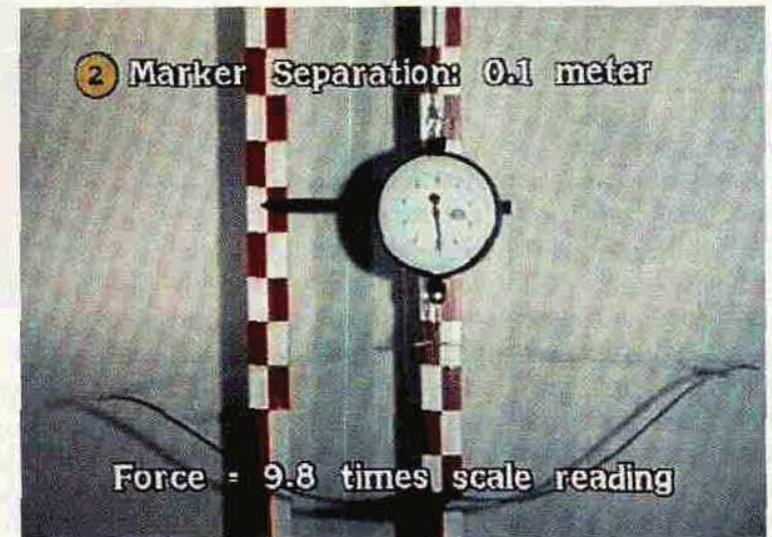

b

An archer shoots an arrow from a compound bow (a). A spring scale is used to measure the force needed to pull back the string of the compound bow a measured distance (b). Students combine data from the two scenes to compare the work done on the arrow with its kinetic energy. Figure 6 

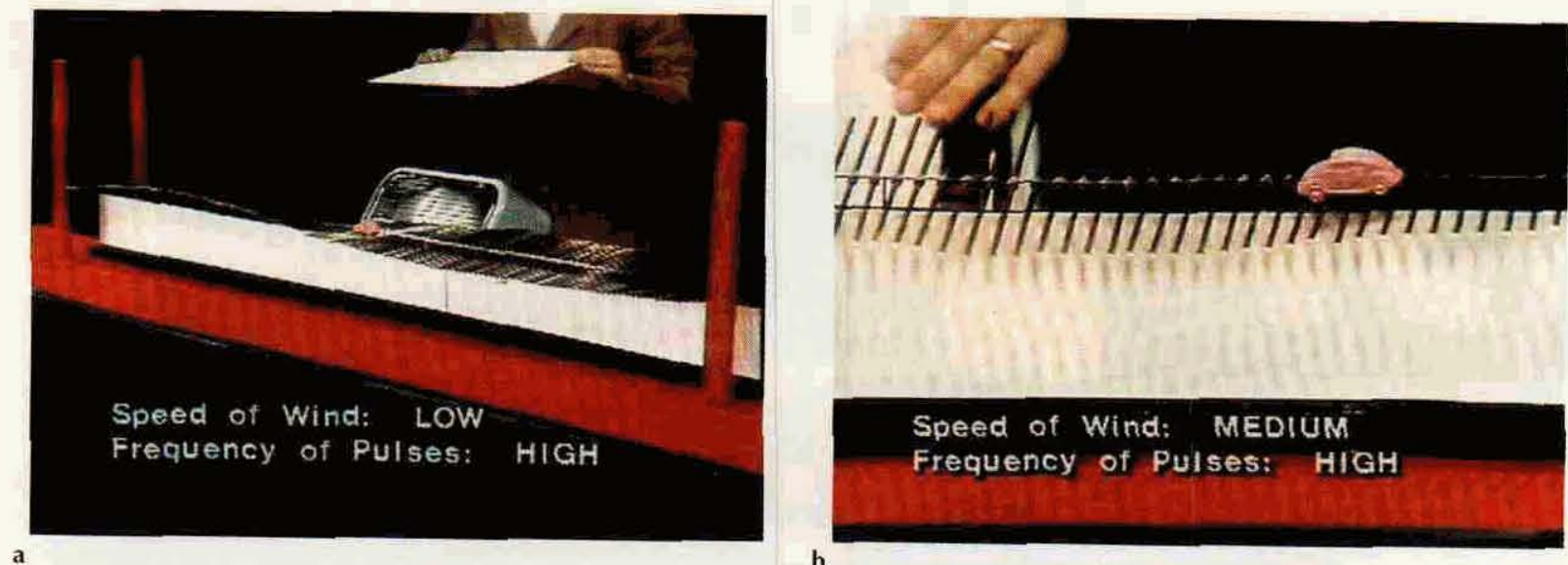

To study the collapse of the Tacoma Narrows Bridge, students can select from different scenes of an electric fan blowing on a model bridge (a). In a close-up (b) they can watch the motion of a toy car on the model bridge. Figure 7

than analog form, all of the image processing techniques for computer graphic images are available. These techniques, often called synthetic video processing, enable the user to combine successive video frames and to play back video from perspectives and in modes different from those used during recording. Because this processing provides students with a visual means to answer, "what if..." questions, it may help students better understand the abstract concepts of physics in terms of their own concrete experiences. ${ }^{19}$

\section{Future prospects}

We began our work in interactive video almost 15 years ago with the conviction that this visual medium provides a significant new pedagogical tool for the physics teacher. Building on Bruner's concept, not only does interactive video offer a way to combine the talents of the film producer and the experienced, knowledgeable physics teacher, but it adds an important ingredient: It gives the control of the learning situation to the classroom teacher and the student. By developing a variety of techniques for interactive video, we and others have offered teachers many ways to use this powerful visualizing medium.

As the medium has matured, we have moved from using acetates on television monitors for simple analyses to using image processing techniques on student experiments captured on video. Driven by the multimedia industry, the hardware and software will continue to improve. The storage capacity and low replication costs of CD-ROM make it easy for teachers to share video scenes and analyses. The developing electronic communication networks offer the prospect of more rapid and convenient exchanges.

The past 15 years of interactive video have been an exciting time in which we have learned much about how to use the medium with students. We anticipate the next few years to be equally exciting, as we and many others explore the capabilities and pedagogical value of emerging video processing techniques, increasingly powerful computers, larger storage devices and electronic communication of video.

Thanks to Christopher J. Moore for collecting the digital images to accompany this article.

\section{References}

1. J. S. Bruner, The Process of Education, Harvard U. P., Cambridge, Mass. (1961).
2. R. G. Fuller, C. R. Lang, eds., Physics: Cinema Classies, videodises produced by Am. Assoc. Phys. Teach., College Park, Md. (1992), distributed by Ztek Co., phone: 800-247-1603.

3. R. G. Fuller, Am. J. Phys. 61, 299 (1993).

4. D. A. Zollman, M. L. Noble, Physics of Sports, videodisc produced by Kansas State U., Manhattan, Kan. (1988), distributed by Video Discovery Inc., phone: 800-548-3472.

5. D. A. Zollman, Physics and Automobile Collisions, videodisc produced by Wiley, New York (1984), distributed by Ztek Co., phone: 800-247-1603.

6. R. G. Fuller, D. A. Zollman, T. C. Campbell, The Puzzle of the Tacoma Narrows Bridge Collapse, videodisc produced by U. of Nebraska, Lincoln, Nebr. (1982), distributed by Ztek Co., phone: 800-247-1603.

7. D. A. Zollman, R. G. Fuller, Energy Transformations Featur ing the Bicycle, videodisc produced by U. of Nebraska, Lincoln, Nebr. (1984), distributed by Ztek Co., phone: 800-247-1603.

8. R. G. Fuller, D. A. Zollman, Studies in Motion, videodise produced by U. of Nebraska, Lincoln, Nebr. (1983), distributed by Ztek Co., phone: 800-247-1603.

9. D. A. Zollman, R. Curtin, M. L. Noble, J. Ed. Tech. Systems 15, 249 (1987).

10. K. Laws, Physics of Dance, Macmillan, New York (1984). A. Armenti, ed., The Physics of Sports, AIP, New York (1992).

11. D. A. Zollman, Phys. Teach. 28, 20 (1990); and in 101 Success Stories of Information Technology in Higher Education, J. Boettcher, ed., McGraw-Hill, New York (1993).

12. D. A. Zollman, R. G. Fuller, Creative Computing, October 1982 , p. 100.

13. See, for example, T. Ducus, J. College Sci. Teaching 23, 166 (1993); S. Hoffmaster, Am. J. Phys. 59, 375 (1991); R. Speers, W. Ploughe, P. Adams, AAPT Announcer 23(4), 38 (1993); J. Edmonds, Phys. Teach. 27, 625 (1989); W. M. MeCord, AAPT Announcer 17(4), 45 (1987); J. R. Sybert, AAPT Announcer 11(4), 79 (1981); D. A. Zollman, Phys. Teach. 18, 395 (1980); D. A. Zollman, G. Athey, Phys. Teach. 14, 178 (1976).

14. R. Beichner, Phys. Teach. 27, 348 (1989); J. Res. Sci. Teaching 27, 803 (1990).

15. L. Winters, N. Lee, S. Allen, E. Smith, Phys. Teach. 31, 376 (1993).

16. R. Dengler, K. Luchner, D. Zollman, Comput. Phys. 7, 393 (1993). R. Dengler, K. Luchner, Eur. J. Phys. 10, 106 (1989). E. Keshishoglow, P. Siegmann, Am. J. Phys. 57, 17 y (1989). E. Huggins, private communication (1988).

17. A. Rubin, Commun. Assoc. Computing Machinery 36(5), 64 (1993).

18. J. Wilson, E. Redish, Comput. Phys, 6, 282 (1992). P. Laws, AAPT Announcer 22(4), 58 (1992). G. Novak, D. Davis, L. MacDonald, R. Fuller E. T. Patterson, AAPT Announcer 23(4), 34 (1993)

19. W. Schanse, R. A. Kolvoord, J. Morrow, AAPT Announcer 23(4), 45 (1989). R. Cadmus Jr., Am. J. Phys. 58, 397 (1990). 